\title{
Posturographic study of human body sway before and after a work day for diagnosis of tiredness
}

\author{
Michail Karpinski ${ }^{1}$, Natalya Kizilova ${ }^{2,3}$ \\ ${ }^{1}$ N. I. Sytenko Institute of Spine and Joint Pathology, Ukraine \\ ${ }^{2}$ Warsaw University of Technology, Poland \\ ${ }^{3}$ Vilnius Gediminas Technical University, Lithuania \\ E-mails: ${ }^{1}$ medicine@online.kharkov.ua,${ }^{2}$ n.kizilova@gmail.com (corresponding author) \\ (Received 11 June 2016; accepted 19 August 2016)
}

\begin{abstract}
Body sway measured by force platform is an excellent simple test for diagnostics of locomotory, balance, visual and neural disorders. In this study the body sway parameters have been measured on a group of healthy medical workers in the morning/evening time before/after their work day. The centre of pressure $(\mathrm{CoP})$ trajectories in a series of different 2-leg stances and during a step forward off from the force platform have been recorded. It was found, in the morning the sway amplitudes were higher due to relaxed state of muscles, while after the work day the muscles were tired and the force platform test demanded more attention and body control which mostly lead to lower body sway amplitudes. The step off from the platform were executed faster in the evening, and the more elderly individuals exhibited more pronounced pathological signs in the $\mathrm{CoP}$ trajectory in the evening. Basing on the data analysis, new indices of tiredness and stress level are proposed for occupational medicine.
\end{abstract}

Keywords: body sway, posture balance, control, medical diagnostics, stress, tiredness.

\section{Introduction}

Postural stability is an important constituent of body health for it is based on the state of the skeletal, muscular, neural, visual, balance systems and their interactions. Computer-assisted posturography is widely used in medical diagnostics and sport medicine as fast, simple and reliable test of human locomotory system. Posturographic curves may be explained within accepted biomechanical models of different complexity, from the inverted pendulum discrete models to the 3D multibody finite element models [1]. The force platform allows measurements of the CoP coordinates $\left(X_{C}(t), Y_{C}(t)\right)$ and computations the CoP trajectory $Y_{C}\left(X_{C}\right)$ at different 2-leg and 1-leg stances [1-3]. Pattern of shifts in the CoP location when the body mass is transferred onto the left/right leg can be used for separation between the spine (osteochondrosis) and joint (coxarthrosis) problems in elderly patients [2]. Here the first experimental evidence of early diagnostics of tiredness and stress state from the posturographic data is presented. In the recent literature the sway parameters of the patients with different locomotory disorders only in the morning time [3] or after the sleepless night [4] could be found. Here the posturography data measured on the same volunteers before and after their 8-hour work day is analysed, that allows quantitative estimation of the normal daily tiredness for the persons of different age.

\section{Methods}

The force platform 'Statograph-6' of the laboratory of biomechanics Kharkov Institute of Spine and Joint pathology has been used. Twenty healthy individuals (weight $71.4 \pm 12.1 \mathrm{~kg}$, height $1.7 \pm 0.2 \mathrm{~m}$, and age $45 \pm 17$ years) without pronounced neuromuscular disorders have been asked to maintain upright stance on the force platform during $30 \mathrm{~s}$. All the volunteers signed a written agreement on usage of their anonymous medical data for research purposes. The age, height, weight, gender and results of the standard series of 2-leg stances reported by authors in the previously published papers [2, 3] have been recorded and analysed (Fig. 1). It was a series of five 
30-s tests included a quite symmetric 2-leg stance (Fig.1a), transfer of the body mass onto the left (Fig. 1b) and right (Fig. 1c) legs followed by a step off from the platform with left and right legs accordingly (Fig. 1d). The measured time series $\left(X_{C}(t), Y_{C}(t)\right)$ have been then amplified and the low $(f<0.01 \mathrm{~Hz})$ and high $(f>10 \mathrm{~Hz})$ frequency components have been subtracted using the second order Butterworth filter. Each of the trajectory $X_{C}\left(Y_{C}\right)$ were restricted by rectangle $\{X \epsilon$ $[-a, a], Y \in[-b, b]\}$, where $a=\left|\max \left(X_{C}\right)-\min \left(X_{C}\right)\right| / 2, b=\left|\max \left(Y_{C}\right)-\min \left(Y_{C}\right)\right| / 2$. The values $2 \mathrm{a}$ and $2 \mathrm{~b}$ have been considered as sway amplitudes in the frontal and sagittal planes accordingly. The trajectories like presented in fig.1e and fig.1f were proper to the younger (age $<45,10$ persons) and the elderly (age > 45) subgroups of the volunteers accordingly.

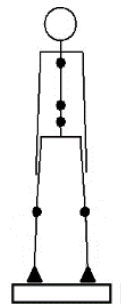

a

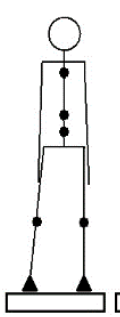

b

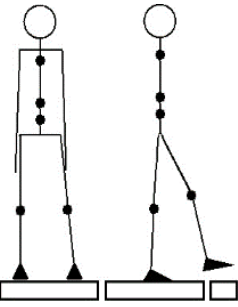

$\mathrm{c}$

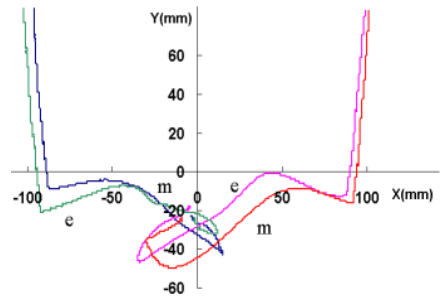

e

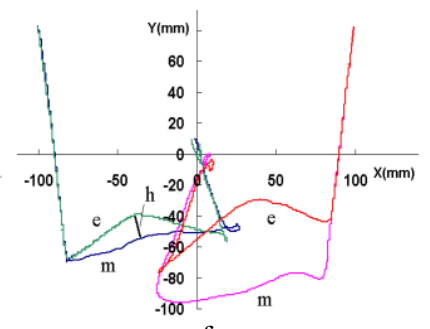

$\mathrm{f}$

Fig. 1. 2-leg stances (a, b, c) and step off from the force platform (d) studied in experiments, and $Y(X)$ trajectories of the first step off towards from the force platform with slight (e) and strong (f) morningevening differences. The morning and evening trajectories are marked by $m$ and $e$

Body sway can be efficiently described by the inverted n-link pendulum model described by Lagrange equations:

$$
\frac{d}{d t} \frac{\partial T(t)}{\partial \dot{\Theta}_{j}}-\frac{\partial T(t)}{\partial \Theta_{j}}+\frac{\partial \Pi(t)}{\partial \Theta_{j}}=q_{j}(t-\tau),
$$

where $T$ and $\Pi$ are kinetic and potential energy, a dot over the symbol represents the time derivative, $\Theta_{j}$ is the angle between the $j$-th segment and vertical axis, $q_{j}$ is the net moment of force in the $j$-th joint, $\tau$ is the time delay caused by reaction of the nervous system to information brought via receptors from the muscles and balance system. The time delay is bigger in elderly persons and at some neural diseases [1]. For each individual his/her body mass and height have been measured and the mass and moments of inertia of the segments of body have been computed from the tables [1]. Then the right-hand side of (1) was directly computed and the time delay in the control functions $q_{j}(t-\tau)$ was found by correlation analysis with the values $\Theta_{j}(t)$ computed from the measurement data.

As it was shown in different studies, generally $q_{j}(t)=F\left(\Theta_{j}, \dot{\Theta}_{j}\right)$, but usually the dependence $q_{j}(t)=k \Theta_{j}$ gives satisfactory results. Our correlation analysis revealed the time delay $\tau_{s, f}$ is slightly different for the sagittal (s) and frontal (f) plane models. The best correspondence to the measured data has been obtained for $q_{j}(t, \tau)=k_{1} \Theta_{j}(t-\tau)+k_{2} \dot{\Theta}_{j}(t-\tau)$.

\section{Results}

Analysis of the $X_{C}\left(Y_{C}\right)$ curves revealed two groups of individuals. The subjects of the first group have not demonstrated any qualitative differences in the trajectories of their CoP (Fig. 1e). Initial location of the $\mathrm{CoP}$ was the same and only slight quantitative differences between the curves have been found. The subjects of the second group demonstrated normal 'healthy' patterns in the 
morning and clear signs of locomotory problems after the work day (Fig. 1f). According to our previous data $[2,3]$, young healthy volunteers transfer their body mass onto their support leg and make the step off with another leg, and the trajectories of their CoP are looking like left and right curves $(m)$ in Fig. 1f. The elderly patients with spine and joint diseases demonstrate trajectories like those marked by $(e)$ in Fig. 1f. During this step off the CoP moves forward, then backward and then again forward producing the 'hill' which 'height' $h$ (Fig. 1f) is a confirmed characteristic of locomotory disorders [3]. It means the tiredness after the work day reveals hidden locomotory pathology that could be efficiently tested by posturographic test.

The tested individuals also demonstrated certain shift in the averaged CoP location in the morning and evening. The dependence $\mathrm{dY}(\mathrm{dX})$ between the shifts in $\mathrm{X}$ and $\mathrm{Y}$ coordinates in three corresponding 2-leg stance tests in the morning and evening is presented in Fig. 2a. Almost uniform shift in different directions (forward-backward, left-right) has been detected. The absolute value of the shift was bigger when in the morning tests the averaged CoP located further from the centre of coordinate system. It means, the body position was more stable when $\mathrm{CoP}$ was projected onto the $(\mathrm{X} 0 \mathrm{Y})$ plane closer to $(0,0)$.

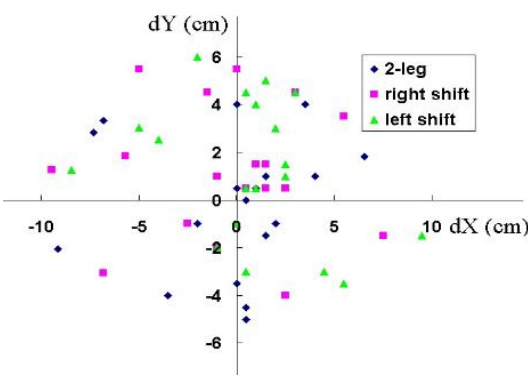

a

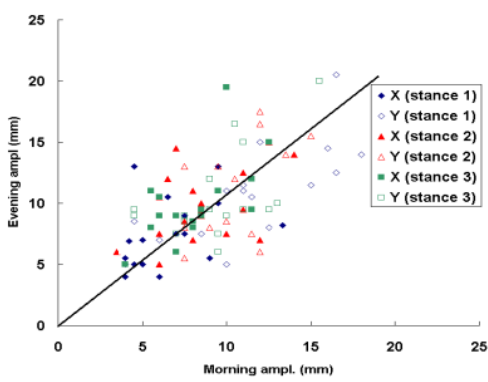

$\mathrm{b}$

Fig. 2. Shift $(\mathrm{dX}, \mathrm{dY})$ in location of the CoP (evening - morning) for different stances

The sway amplitudes could be either bigger (the marks over the straight line in Fig. 2b) or lower (the marks below the straight line in Fig. 2b), while the absolute variation in the amplitudes is smaller for the sway in the frontal plane (filled in marks in Fig. 2b) than the sway in the sagittal plane (empty marks in Fig. 2b). Since increase of the frontal sway is a good predictor of sudden fall in elderly persons [1], the test of tiredness could be a good diagnostic tool for early diagnostics of neural and other balance control systems.

\section{Conclusions}

It was shown the lower body sway amplitude and faster step off from the force platform are the important signs of tiredness after a work day which are proper to the elderly individuals. As it was shown before, those signs are also proper to the patients with locomotory disorders, independently on the hours of examination. The n-link model of the inverted pendulum with the delayed control function allows evaluation of the time delay $\tau$ between the generalized coordinates $\Theta_{j}(t)$ and the functions $q_{j}$ controlling the body stability. The time delay may be proposed as a new index of daily tiredness and stress level in occupational medicine.

\section{References}

[1] Latash, M. L.; Zatsiorsky, V. M. [eds.] 2001. Classics in Movement Sciences. Champaign: Human Kinetics. 464 p.

[2] Kizilova, N., et al. 2009. Posturographic study of the human body vibrations for clinical diagnostics of the spine and joint pathology, Mechanika 6(60): 37-41. 
[3] Sørensen, R. R., et al. 2014. Impaired postural balance in the morning in patients with knee osteoarthritis, Gait \& Posture 39(4): 1040-1044. http://dx.doi.org/10.1016/j.gaitpost.2014.01.002

[4] Fabbri, M., et al. 2006. Postural control after a night without sleep, Neuropsychologia 44(12): 25202525. http://dx.doi.org/10.1016/j.neuropsychologia.2006.03.033 